\title{
Experimental Investigation on Jets issuing from Angular Cut Nozzles
}

\author{
Thanigaiarasu. $\mathrm{S}^{1}$, Divvela Rakesh ${ }^{2}$, Vishnu Raj ${ }^{3}$, Manikandan. $\mathrm{S}^{4}$, Elangovan. $\mathrm{K}^{5}$ and Lovaraju.P $\mathrm{P}^{6}$ \\ ${ }^{1}$ Department of Aerospace Engineering, Anna University, Chennai, India \\ ${ }^{2}$ Department of Aerospace Engineering, Lakireddy Balireddy College of Engineering, Mylavaram, India
}

\begin{abstract}
The intention of this experimental study is to investigate the external flow behavior through angular cut nozzles where the exit area transforms from circular to non-circular by making a cut at the exit plane of the nozzle with a particular angle. This study helps in understanding the enhancement of jet mixing characteristics by observing the CMD which gives an idea about the change in Mach number along the axial direction. The analysis were carried out for 4 models whose angular cut at the exit plane was $5^{\circ}, 10^{\circ}, 15^{\circ}$ and $20^{\circ}$ respectively an operated at three different NPR i.e. at 1.2, 1.4 and 1.6 and the results that are obtained is compared with the circular nozzle and the results justifies that there is an augmentation in jet mixing with increase in the degree of cut and the maximum advancement in jet mixing exists at $20^{\circ}$ degree cut.
\end{abstract}

Keywords: Angular cut nozzle, Jet mixing, Mach number

\section{Introduction}

There are many schemes for energizing the jet mixing property i.e. when the flow enters into the ambient atmosphere by applying the techniques like co-flowing jets and installing of jet tabs at the exit plane will enhance the jet mixing character by creating turbulence with developing vortices in the free-stream jet at a cost of slight reduction in the efficiency of the nozzle. Although there is a reduction in efficiency jet mixing plays a prominent role in combustion, noise reduction and also serves in many other applications. The researches[1-6] even carried out in the bevel nozzles and notched circular nozzles in which effective jet mixing has taken place. They also specified that the mixing properties also depend upon the aspect ratio of the nozzle. Even though researches were carried out in the field of jet mixing, but a very few researches were carried out in angular cut nozzles, in that it had been considered as a comparative model and to other nozzles and observed their performances. But, no one had made any qualitative analysis in the angular cut nozzles. So, the present investigation focuses on the qualitative and quantitative aspects regarding the jet mixing properties on angular cut nozzles, whose exit plane was trimmed at angles $5^{\circ}, 10^{\circ}, 15^{\circ}$ and $20^{\circ}$, and observed the centreline Mach number decay in comparison with the circular convergent nozzle. This study helps in better understanding regarding the promotion of the jet mixing properties with increment in angle of attack.

\section{Experimental Procedure}

\subsection{Experimental setup}

The experiments have been conducted in the "High Speed Jet Laboratory" with an operating range of 0-8 bar. The schematic diagram of the experimental setup. The pressure ratio is to be maintained i.e. total pressure in the stagnation chamber to atmospheric pressure with for the corresponding Mach number. At present the nozzle is operated at NPR 1.4 and 1.6 whose corresponding Mach numbers are 0.7 and 0.85 respectively. This Pitot tube is connected to a traverse mechanism which helps to move the Pitot tube along $\mathrm{X}, \mathrm{Y}$ and $\mathrm{Z}$ directions that helps to calculate the pressure decay along the axial direction and radial direction. 


\subsection{Nozzle Design}

The nozzles that are used for experimental work had been fabricated by using brass material for superior finishing of surface which helps to reduction in internal flow dissimilarities as well as the control in the boundary layer formation at high speed operational conditions. The dimensions of the nozzle are, the semi cone angle is $10^{\circ}$ and the length of the nozzle is $100 \mathrm{~mm}$, the slant length of the nozzle is $101.543 \mathrm{~mm}$ and the inlet diameter of the nozzle is $55.26 \mathrm{~mm}$ and the exit diameter of the nozzle is maintained at $20 \mathrm{~mm}$. the thickness of the nozzle wall is maintained at $2 \mathrm{~mm}$. It consists of an extended threading length of $15 \mathrm{~mm}$ which helps to connect to the base plate.
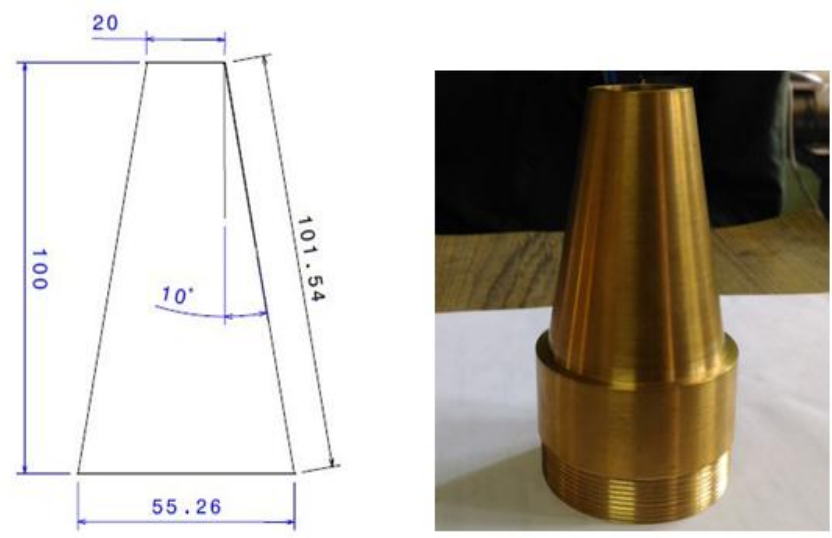

Fig.1: Dimensional view and photographic view of circular nozzle

When the nozzle is consisting an angular cut at the exit plane the dimensions of the nozzle will be partially changes. The percentage change in slant length of the nozzle due to angular cut is, for a $5^{0}$ degree angular cut the change in slant length of the nozzle is $1.77 \%$.In the case of $10^{\circ}$ degree angular cut the change in slant length of the nozzle is determined to be $3.64 \%$.The final dimensions of the nozzle after having a slant cut of $10^{\circ}$ degrees. In the case of $15^{\circ}$ degree cut the change in slant length is obtained as $5.63 \%$. Finally, in the case of $20^{\circ}$ degree cut the change in slant length of the nozzle is very higher i.e. about $7.77 \%$. This percentage decrease in slant length of the nozzle leads to the transition of flow behaviour in nozzle. The nozzle is not directly connected to the pocket, to fix the nozzle to the stagnation chamber a base plate have been designed which facilitates to connect all the angular cut nozzles to the stagnation chamber. The dimensions of the designed base plate is $59.26 \mathrm{~mm}$ inner diameter and the outer diameter of the plate is $100 \mathrm{~mm}$

\section{Results and Discussion}

The investigation had been carried out on the high speed jets in circular convergent nozzles, which were operated at NPR 1.2, 1.4 and 1.6 The analysis has been done on centerline Mach number decay along the axial direction to understand the behavior of the flow. The results have been expressed with respect to nondimensional Mach number (M/Me) and axial direction(X/D). It is observed that the potential core length of the circular nozzle is higher than the 5 degree angular cut nozzle. The potential core length of the circular nozzle is extended upto $\mathrm{X} / \mathrm{D}=5.4$ and then it starts decaying continuously along the axial distance as shown in the Fig2.0. But, in case of 5 degree angular cut the potential core length is extended upto $X / D=4.8$ and then it starts decaying. The percentage decrement in the potential core length due to 5 degree angular cut is approximately $12 \%$.Figure 3.0 shows the jet decay along the centerline for 10 degree angular cut nozzle when compared with the circular nozzle. 


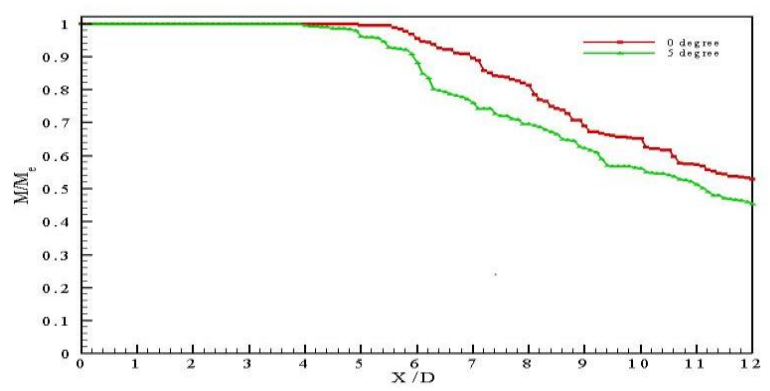

Fig.2: CMD profile for 5 degree angular cut nozzle at NPR1.2

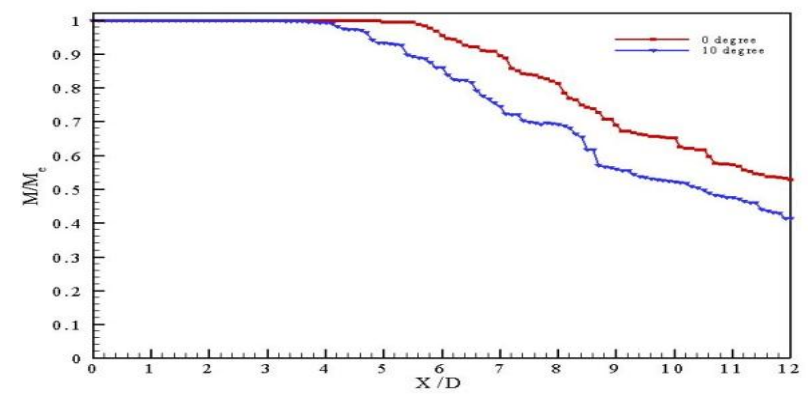

Fig.3: CMD profile for 10 degree angular cut nozzle at NPR1.2

The potential core length of the 10 degree angular cut nozzle is extended upto $X / D=4.3$ and then it gradually decays along the axial direction. As it was observed in Fig.3.0that the potential core length of the circular nozzle is extended upto $\mathrm{X} / \mathrm{D}=5$.4. So, the percentage reduction in the potential core length along the centerline due to the 10 degree angular cut is $20.4 \%$..Figure 4.0 shows the jet decay along the centerline for 15 degree angular cut nozzle when compared with the circular nozzle.

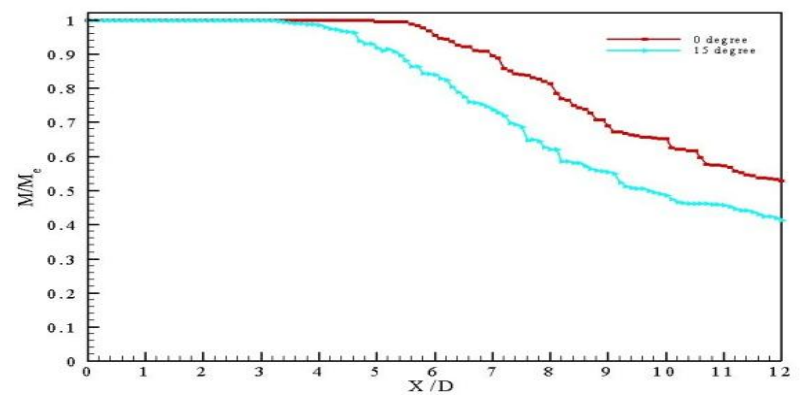

Fig.4: CMD profile for 15 degree angular cut nozzle at NPR1.2

The previous graph shows the jet decay along the centerline for 15 degree angular cut nozzle when compared with the circular nozzle. The potential core length of the 15 degree angular cut nozzle is extended upto $\mathrm{X} / \mathrm{D}=3.7$ and then it gradually decays along the axial direction. As it was observed that the potential core length of the circular nozzle is extended upto $\mathrm{X} / \mathrm{D}=5.4$.So, the percentage reduction in the potential core length along the centerline due to the 15 degree angular cut is $31.4 \%$. The following graph shows the jet decay along the centerline for 20 degree angular cut nozzle when compared with the circular nozzle. 


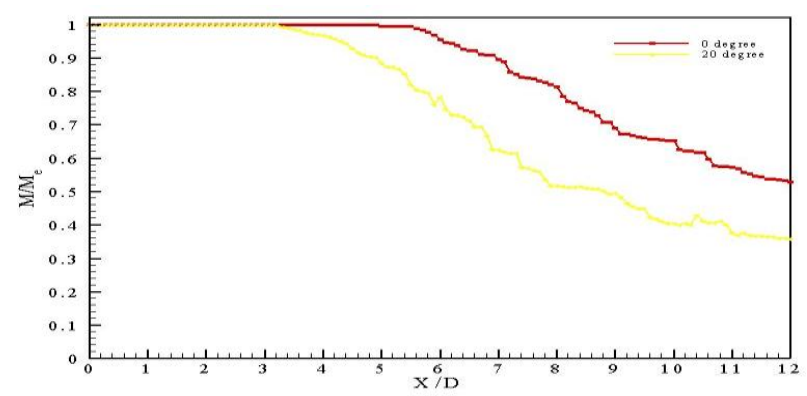

Fig5: CMD profile for 20 degree angular cut nozzle at NPR1.2

The potential core length of the 20 degree angular cut nozzle is extended upto $\mathrm{X} / \mathrm{D}=3.4$ and then it gradually decays along the axial direction. As it was observed that the potential core length of the circular nozzle is extended upto $\mathrm{X} / \mathrm{D}=5.4$.So, the percentage reduction in the potential core length along the centerline due to the 20 degree angular cut is $37.04 \%$ as shown in Figure 5.0.The following centerline Mach number decay profiles clearly explain the jet decay along the axial direction in comparison with the circular nozzle for NPR=1.4.

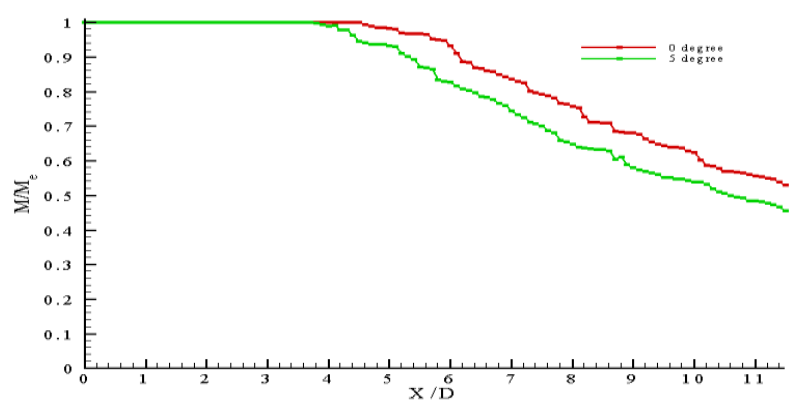

Fig.6: CMD profile for 5 degree angular cut nozzle at NPR1.4

In the above centerline Mach number profile it was observed that the potential core length for a circular nozzle is extended upto $\mathrm{X} / \mathrm{D}=4.8$ (Fig.6.0) and then it starts decreasing continuously along the axial direction. But, for 5 degree angular cut it was observed that the potential core length is extended upto X/D = 4.2 and then it starts decreasing continuously along the axial direction. The percentage decrease in the potential core length due to the presence of 5 degree angular cut is $12.5 \%$. The succeeding graph explains about the decay along the centerline Mach number for 10 degree angular cut. In the previous graph, the centerline Mach number profile for a circular nozzle had been observed, it states that the potential core length is extended upto X/D $=4.8$ and then it starts decreasing continuously along the axial direction. But, for 10 degree angular cut nozzle it was observed from the figure 7.0 that the potential core length is extended up to $\mathrm{X} / \mathrm{D}=3.8$ and then there is a continuous decay in Mach number along the axial direction. The percentage decrease in the potential core length due to the presence of 10 degree angular cut is $20.8 \%$. The following centerline Mach number decay profile clearly explains the jet decay along the axial direction for 15 degree angular cut nozzle as shown in the Fig.8.0 in comparison with the circular nozzle for NPR=1.4. In the above centerline Mach number profile it was observed that the potential core length for a circular nozzle is extended upto $\mathrm{X} / \mathrm{D}=5.4$ and then it starts decreasing continuously along the axial direction. But, for 10 degree angular cut it was observed that the potential core length is extended upto $\mathrm{X} / \mathrm{D}=3.8$ and then it starts decreasing continuously along the axial direction. 


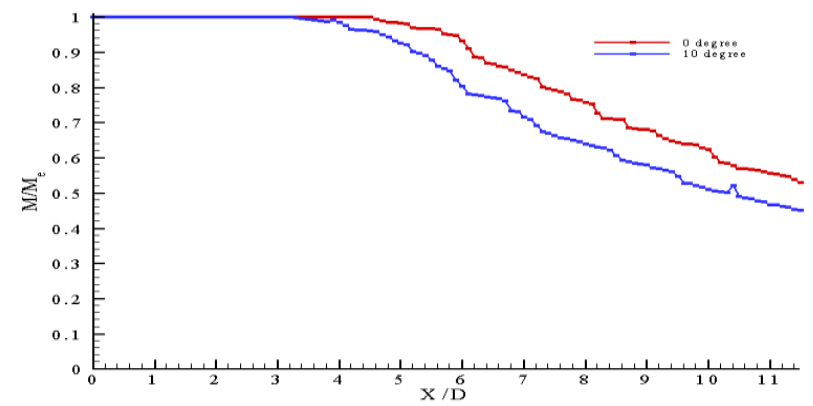

Fig.7: CMD profile for 10 degree angular cut nozzle at NPR1.4

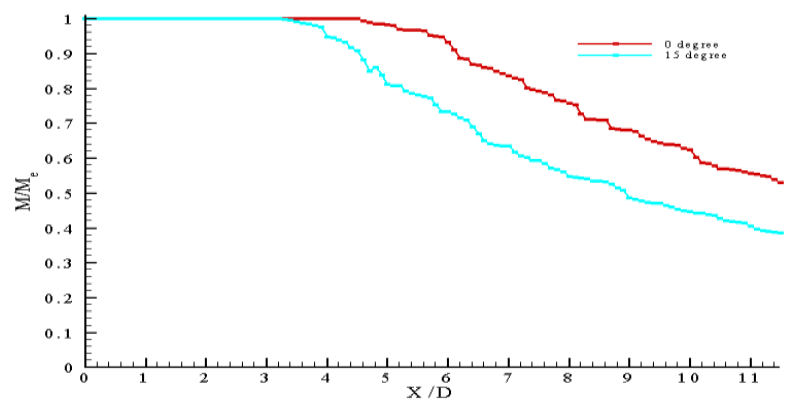

Fig8: CMD profile for 15 degree angular cut nozzle at NPR1.4

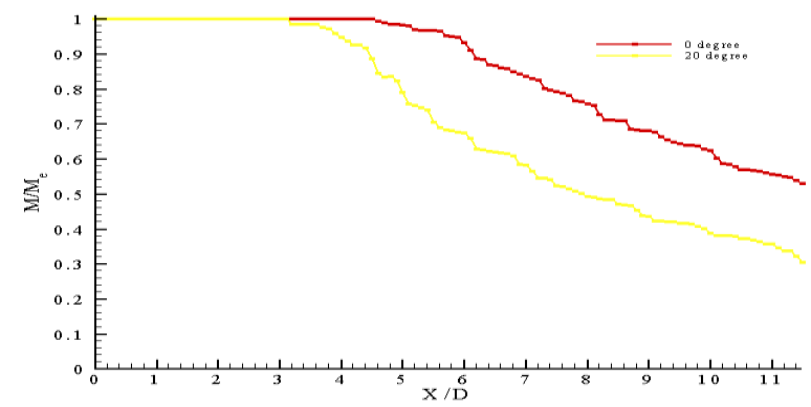

Fig 9: CMD profile for 20 degree angular cut nozzle at NPR1.4

In the above centerline Mach number profile it was observed that the potential core length for a circular nozzle is extended upto X/D $=4.8$ and then it starts decreasing continuously along the axial direction. But, for 20 degree angular cut it was observed from Figure 9.0that the potential core length is extended upto X/D =3.1 and then it starts decreasing continuously along the axial direction. The percentage decrease in the potential core length due to the presence of 20 degree angular cut is 35.4\%.The following centerline Mach number decay profiles explains about the jet decay along the axial direction in comparison with the circular nozzle for $\mathrm{NPR}=1.6$. The following centerline Mach number decay profile clearly explains the jet decay along the axial direction for 5 degree angular cut nozzle in comparison with the circular nozzle for NPR=1.6. In the above centerline Mach number profile it was observed that the potential core length for a circular nozzle is extended upto $\mathrm{X} / \mathrm{D}=5.4$ and then it starts decreasing continuously along the axial direction. But, for 5 degree angular cut it was observed from Fig.10.0 that the potential core length is extended upto X/D =4.4 and then it starts decreasing continuously along the axial direction. The percentage decrease in the potential core length due to the presence of 5 degree angular cut is $18.5 \%$. The following centerline Mach number decay profile clearly explains the jet decay along the axial direction for 10 degree angular cut nozzle in comparison with the circular nozzle for NPR=1.6 as shown in Figure 11.0. 


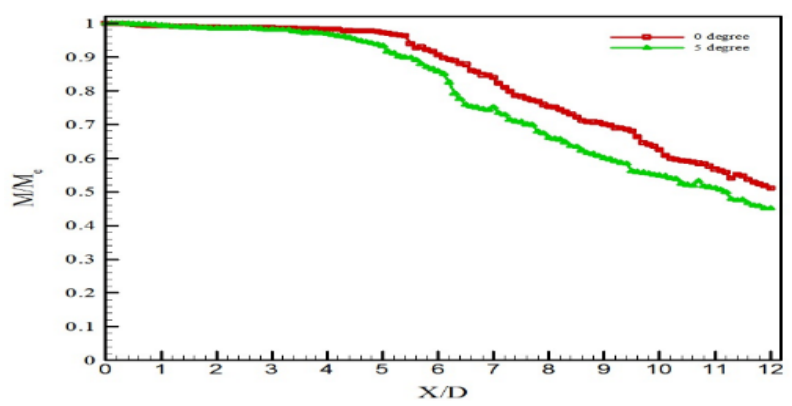

Fig 10: CMD profile for 5 degree angular cut nozzle at NPR1.6

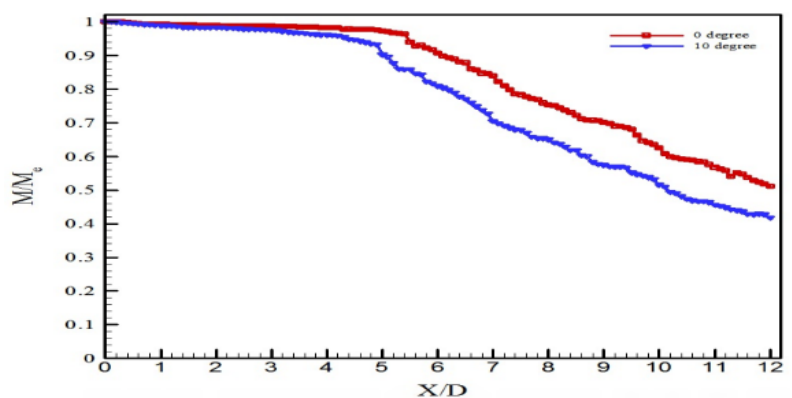

Fig.11: CMD profile for 10 degree angular cut nozzle at NPR1.6

The percentage decrease in the potential core length due to the presence of 10 degree angular cut is 29.6\%.The following centerline Mach number decay profile clearly explains the jet decay along the axial direction for 15 degree angular cut nozzle in comparison with the circular nozzle for NPR=1.6.

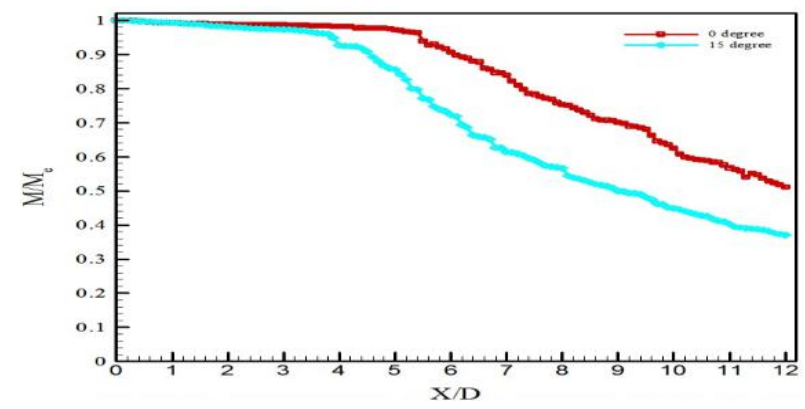

Fig 12: CMD profile for 15 degree angular cut nozzle at NPR1.6

In the above centerline Mach number profile it was observed that the potential core length for a circular nozzle is extended upto X/D = 5.4 and then it starts decreasing continuously along the axial direction. But, for 15 degree angular cut it was observed from Figure 12.0 that the potential core length is extended upto X/D =3.2 and then it starts decreasing continuously along the axial direction. The percentage decrease in the potential core length due to the presence of 15 degree angular cut is $40.7 \%$. The following centerline

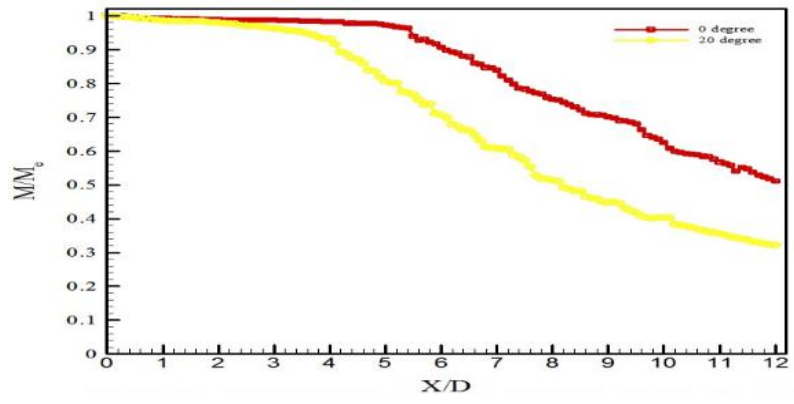

Fig.13: CMD profile for 20 degree angular cut nozzle at NPR1.6 
Mach number decay profile clearly explains the jet decay along the axial direction for 20 degree angular cut nozzle in comparison with the circular nozzle for $\mathrm{NPR}=1.6$. In the above centerline Mach number profile it was observed that the potential core length for a circular nozzle is extended upto X/D $=5.4$ and then it starts decreasing continuously along the axial direction. But, for 20 degree angular cut it was observed from from Fig.13.0 that the potential core length is extended upto $\mathrm{X} / \mathrm{D}=2.8$ and then it starts decreasing continuously along the axial direction. The percentage decrease in the potential core length due to the presence of 20 degree angular cut is $48.1 \%$.

\section{Conclusion}

It had been observed from the centerline Mach number profiles that there is an increment in the jet mixing property by making an angular cut at the exit plane of the nozzle and also it had been proved that there is an increment in the jet mixing with an increase in the cut at the exit plane. As the analysis proves that due to the presence of the angular cut at the exit plane the mixing is directly proportional to the increment in the angle and also it is directly proportional to the exit Mach number of the nozzle. The maximum mixing had been taken place at 200 degree angular nozzle at an NPR 1.6.

\section{Acknowledgment}

I acknowledge and many thanks to the Centre for Technology Development and Transfer (CTDT) for their encouragement and providing funding to do this project in the college campus.

\section{References}

[1] W.G. Hill, P.R. Greene, "Increased turbulent jet mixing rates obtained by self acoustic oscillations," Transactions of the ASME, Journal of Fluids Engineering, vol. 99, pp. 520-525, 1977.

[2] M.A.Z. Hasan, A.K.M.F. Hussain, "The self-excited axisymmetric jet," Journal of Fluid Mechanics, vol. 115, pp. 59-89, 1982

[3] A.K.M.F. Hussain, M.A.Z. Hasan, “The 'whistler-nozzle' phenomena," Journal of Fluid Mechanics, vol. 134, pp. 431-458, 1983.

[4] T.H. New, K.S. Tan, H.M. Tsai, "Effects of non-circular collars on an axisymmetric jet," Physics of Fluids, vol. 19,2007

[5] J. Mi, G.J. Nathan and R.E. Luxton, "Mixing characteristics of a flapping jet from a self-exciting nozzle," Flow Turbulence and Combustion, vol. 67, pp.1 -23, 2001.

[6] G.J. Nathan, S.J. Hill and R.E. Luxton, “An axisymmetric 'fluidic' nozzle to generate jet precession," Journal of Fluid Mechanics, vol.370, pp.347-380, 1998. 